\title{
A certain concentration of vecuronium and pipecuronium can inhibit the expression of neutrophils CD11b in human isolated venous blood
}

Zunyuan Liu ( $\square$ ly29.love@163.com )

\section{Research article}

Keywords: Vecuronium, pipecuronium, CD11b

Posted Date: March 9th, 2020

DOI: https://doi.org/10.21203/rs.3.rs-16446/v1

License: (c) (i) This work is licensed under a Creative Commons Attribution 4.0 International License.

Read Full License 


\section{Abstract}

Background Perioperative medicines can affect the body's immune response, according to data reported a variety of anesthetic drugs can directly or indirectly affect the immune response, this study aims to preliminarily common muscle relaxant presence of anti-inflammatory effects.

Methods To collect peripheral blood of healthy adults, and designed the following treatment groups: [A] blank group; [B] static and unstimulated drug groups: the group contained two concentrations of both pipecuronium and vecuronium respectively $(0.5 / 0.1 \mathrm{~g} \cdot \mathrm{ml}-1)$; [L] simple lipopolysaccharide (LPS) group; [C] under stimulated drug groups: two concentrations $(0.5 / 0.1 \mathrm{~g} \cdot \mathrm{ml}-1)$ muscle relaxants were added with lipopolysaccharide in each group. Detection of average fluorescence emphasized expression of CD11b on neutrophils by flow cytometry.

Results Compared with the blank control group [A], the expression of CD11b in each group was significantly increased in the LPS group [L] and the stimulus drug groups $[C](p<0.001)$. Compared with the blank control group [A], low concentration of vecuronium and pipecuronium could inhibit the expression of CD $11 \mathrm{~b}$ on the surface of neutrophils (vecuronium $95 \% \mathrm{Cl}$ : 20.47 to $37.28, p<0.001$ ),(pipecuronium $95 \%$ $\mathrm{Cl}: 18.63$ to $50.22, p<0.001)$. High concentrations of vecuronium and pipecuronium to CD11b expression differences had no statistical significance $(p>0.05)$.

Conclusions The concentration of vecuronium and pipecuronium that approximates clinical maintenance dose has effect of inhibiting the CD11b expression of human neutrophil in venous blood in vitro. The results of this study can provide the reference for muscle relaxant immunological research and clinical rational use.

\section{Background}

The repeated use of muscle relaxants may have a certain effect on the immune function of the body in the long-term high dose of clinical perioperative period. The drug muscle relaxant can affect the neuroendocrine system by acting on the nerve muscle conduction pathway, which indirectly affects the immune response of the whole organism. In addition, anesthetic muscle relaxants can also directly affect immune cells, regulate the generation of various active factors and affect immune function.

The expression of CD11b on PMN surface plays an important regulatory role in inflammatory response. At present, there are many literatures about the effect of anesthetics on neutrophils, and the effect of muscle relaxant on neutrophils is relatively rare. Krumholz $\mathrm{W}$ observed that pancuronium can effectively inhibit neutrophils adhesion, inhibit neutrophils' respiratory burst, reduce the body's ability to defend against bacteria, and may increase the risk of infection. ${ }^{[1]}$

The study was carried out in healthy adult veins, because the whole blood contains various cytokines and the internal environment of various immune cells, which can lay a foundation for further clinical study. The two concentrations of muscle relaxants in the experiment were also basically close to anesthesia 
induction and maintenance concentration. ${ }^{[2]}$ The expression of CD11b was determined by flow cytometry. In this study, the effects of vecuronium and pipecuronium on the expression of CD11b on neutrophils were analyzed by specific test observations.

\section{Methods}

This experiment was conducted under the approval of medical ethics committee of Liaocheng people's hospital, China. All volunteers received informed consent.

1.

Study Design and Participants

(1). Test materials and test instruments

Pipecuronium (Hungary geely big pharma, Hungary) and vecuronium (Zhejiang Xianju Pharmaceutical Co.Ltd, China) and lipopolysaccharide (Sigma company, USA), FITC tag Mouse Anti - Human CD11b antibodies (Beijing four are cypress biological technology Co. Ltd, China), red blood cell cracking liquid (BD company, USA), PBS (BD company, USA). FACSCalibur flow cytometry instrument (Becton Dicknson company, USA), XW - 80 - a vortex mixer (Shanghai secco industrial co. LTD, China), Adjustable quantitative fluid accumulator (refinement glass instrument plant in Shanghai, China), LD5-2 a type low speed centrifuge (medical centrifuge factory in Beijing, China), thermostatic temperature box (medical thermostatic equipment factory in Shanghai, China).

(2). Test group

Sixteen volunteers, male and female, aged $20 \sim 40$ years, weighed $55 \sim 75 \mathrm{~kg}$ respectively, ASA level I , had no smoking history, no infection and no immune system diseases. Venous blood from elbow was collected from the above volunteers in the morning, and each blood sample was divided into 10 parts on average. The experiment was divided into 10 groups : [A] blank group; [B] static and unstimulated drug groups: the group contained two concentrations of both pipecuronium and vecuronium respectively $\left(0.5 / 0.1 \mu \mathrm{g} \cdot \mathrm{ml}^{-1}\right)$; [L] simple lipopolysaccharide (LPS) group; [C] under stimulated drug groups : two concentrations $\left(0.5 / 0.1 \mu \mathrm{g} \cdot \mathrm{ml}^{-1}\right)$ muscle relaxants were added with lipopolysaccharide (LPS) in each group.

2.

Test steps

(1). Drug preparation and whole blood preparation

BBoth pipecuronium and vecuronium bromide are dissolved in saline. Each drug was assigned to two final concentrations: close to clinical anesthesia induction of muscle relaxant concentration $\left(0.5 \mu \mathrm{g} \cdot \mathrm{ml}^{-1}\right)$ and clinical anesthesia maintain of muscle relaxant concentration $\left(0.1 \mu \mathrm{g} \cdot \mathrm{ml}^{-1}\right)$. The subjects' total 
elbow vein blood was collected from fasting in the morning, and the anticoagulant containing EDTAK2 was added to use the negative pressure blood collector at one time.

,0.02M PBS solution ( $\mathrm{pH}=7.4) ; 0.2 \mathrm{~g} \mathrm{KH} 2 \mathrm{PO} 4 \cdot 2 \mathrm{H} 2 \mathrm{O} ; 2.9 \mathrm{~g} \mathrm{Na} 2 \mathrm{HPO} 4 \cdot 12 \mathrm{H} 2 \mathrm{O} \otimes 0.2 \mathrm{~g} \mathrm{KCl} \otimes 8 \mathrm{~g} \mathrm{NaCl}$,

fthe above-mentioned material dissolved in $950 \mathrm{ml}$ of distilled water, the capacity to $1000 \mathrm{ml}, 4^{\circ} \mathrm{C}$. The whole blood of the elbow vein of fasting volunteers was collected in the morning, and the anticoagulant containing EDTAK2 was added to use the negative pressure blood extractor in one time.

(2). Test process

Each blood sample was distributed to the above treatment group at the same time. In each group, $100 \mathrm{ul}$ blood samples were added to each group. Then, in the blank group [A] and the LPS group [L], the physiological saline was added for a control of $100 \mathrm{ul}$. In the rest groups, the corresponding final concentration was achieved by adding the corresponding concentration of the drug 100 ul. Put the above each sample in $37^{\circ} \mathrm{C}$ constant temperature box away from light incubation 1.5 hour. [L] and [C] in each group, lipopolysaccharide $100 \mathrm{ul}$ with a final concentration of $1 \mu \mathrm{g} \cdot \mathrm{ml}^{-1}$ was added. In the blank group [A] and the drug groups with no stimulation $[\mathrm{B}]$, a physiological saline $100 \mathrm{ul}$ was added for comparison. Each sample to continue into the $37^{\circ} \mathrm{C}$ constant temperature box away from light incubation 2 hour. Each sample to join to the end of $5 \mathrm{ml}$ fluid tube, take $10 \mathrm{ul} \mathrm{FITC} \mathrm{(fluorescein} \mathrm{isothiocyanate)} \mathrm{tag} \mathrm{CD11b}$ antibodies to join, pipe flow and mixed at room temperature $25^{\circ} \mathrm{C}$ avoid light incubation 20 minutes; Add $2 \mathrm{ml}$ erythrocyte lysate and mix it on the vortex mixer. Leave it for 10 minutes to dissolve the erythrocyte. The sample was placed in the centrifuge and centrifuged at $1000 \mathrm{rpm}$ for 5 minutes. The cells were resuspended by adding $2 \mathrm{ml}$ PBS lotion, and then the samples were placed into the centrifuge for 5 minutes at $1000 \mathrm{rpm}$. The cells were resuspended with $0.5 \mathrm{ml}$ PBS solution. In addition, each sample was provided with a tube of negative control, and FITC (fluorescein isothiocyanate) was used to mark the same type of matching mouse igg $^{-1}$ monoclonal antibody into the blood as a negative control, and the experimental process was the same as above. Finally, the samples were detected by flow cytometry on the computer. ${ }^{[3,4]}$

3.

Test analysis

The flow cytometer was set to detect 5,000 cells, with the excitation wavelength of $488 \mathrm{~nm}$ and emission wavelength of $525 \mathrm{~nm}$. When measured, PMN, monocytes and lymphocytes were separated by forword scatter and side scatter, and the threshold was set as 200 forward scattering measurement to exclude cell debris. CD11b expression level was expressed by mean fluorescence intensity (MCF) on PMN, and analyzed by CELLQuest software. Effects of samples in each group on the average optical density intensity of cd11b expression (Table.1). Optical density intensity of CD11b expression in isolated venous blood cells under resting and unstimulated conditions $\square$ Figure. 1区. Expression of neutrophils $\mathrm{cd} 11 \mathrm{~b}$ in isolated venous blood of resting and unstimulated groupDFigure.2区. Expression of neutrophils CD11b in isolated venous blood of low concentration vecuronium group without stimulation $\square$ Figure. $3 \otimes$. Expression 
of neutrophils CD11b in isolated venous blood of low concentration pipecuronium group without stimulation $\square$ Figure. $4 \rrbracket$.

\section{Statistical Analysis}

Statistical software SPSS 18.0 was used for rank-sum test, Friedman test and measurement data were indicated. $\mathrm{P}<0.05$ was considered statistically significant.

\section{Results}

Compared with the blank control group [A], the expression of CD11b in each group was significantly increased in the LPS group [L] and the stimulus drug groups [C]. Compared with the blank control group $[A]$, low concentration of vecuronium and pipecuronium could inhibit the expression of CD11b on the surface of neutrophils (vecuronium 95\% Cl: 20.47 to $37.28, \mathrm{p}<0.001$ ),(pipecuronium 95\% Cl: 18.63 to $50.22, p<0.001)$. High concentrations of vecuronium and pipecuronium to CD11b expression differences had no statistical significance $(p>0.05)$. The expression of CD11b in vecuronium low concentration group were lower than its high group ( $95 \% \mathrm{Cl}:-110.47$ to $-16.31, \mathrm{p}=0.01$ ). The expression of $\mathrm{CD} 11 \mathrm{~b}$ in pipecuronium low concentration group was lower than its high group ( $95 \% \mathrm{Cl}:-60.32$ to $-23.26, \mathrm{p}<$ 0.001). The rest of the comparative differences between each group were no statistical significance.

\section{Discussion}

It was found and analyzed that both pipecuronium and vecuronium could inhibit the expression of cd11b on neutrophil surface when the concentration of pipecuronium and vecuronium were close to the clinical maintenance concentration (low concentration of the test group). The expression of cd11b was not affected by the concentration of pipecuronium and vecuronium. Lipopolysaccharide could significantly activate the expression of $c d 11 \mathrm{~b}$ on neutrophil surface. Vecuronium and pipecuronium did not affect the expression of cd11b induced by lipopolysaccharide activation on neutrophils. The whole blood sample was used and the incubation temperature was kept at $37^{\circ} \mathrm{C}$, which was close to the physiological internal environment of the organism as far as possible, so as to avoid the damage of the physiological internal environment caused by the pure separation of neutrophils, which resulted in the decrease of the activity of the isolated neutrophils. The whole blood pattern can effectively maintain the physiological activity of neutrophils. ${ }^{[5]}$ It provides a good reference for further clinical trials in vivo. However, all kinds of immune cells and various immune factors in whole blood may be involved in regulating the expression of cd11b, and the whole blood environment may also affect the correctness of the conclusions. Krouholz w et al studied the effect of pancuronium bromide on the adhesion of neutrophils through in vitro experiments. ${ }^{[6]}$ They were $1.3 \mu \mathrm{g} \cdot \mathrm{ml}^{-1}$ and $0.5 \mu \mathrm{g} \cdot \mathrm{ml}^{-1}$ respectively. It was found that both clinical concentrations of pancuronium could directly affect neutrophil adhesion to neutrophil inhibition and significantly affect the chemotaxis of neutrophils to inflammatory sites. The inhibitory effect of high concentration of pancuronium was stronger. However, low concentration of vecuronium and pipecuronium could effectively inhibit the expression of adhesion molecule cd11b on neutrophil surface, but the high 
concentration of drug had no effect on the expression of adhesion molecule on neutrophil surface. This is different from the conclusion of krumholz $\mathrm{w}$ et al. It is speculated that the effect of different drugs on neutrophil function is not concentration dependent, or there are many other influencing factors or mechanisms. In this study, three kinds of muscle relaxants used high and low concentrations, close to the clinical induction concentration and muscle relaxant maintenance concentration respectively, as to whether the other concentrations of drugs can reach statistically significant conclusions need to be further experimental proof. In this paper, the random block design of the same blood sample is used to reduce the test error effectively. Due to the use of small sample data in the experiment, the exact conclusion remains to be demonstrated by repeated tests of multiple concentrations in large samples.

Known some anesthetics have certain inhibition of neutrophil respiratory burst, Muhling $\mathrm{J}$ et al found midazolam can change neutrophils in the free amino acids and alpha keto acid content, and significantly reduce the immune function index superoxide anion (02) the formation of hydrogen peroxide ( $\mathrm{H} 2 \mathrm{O} 2)$, and release MOP (myeloperoxidase, myeloperoxidase), midazolam dose dependent decrease peripheral blood neutrophils macrophages breathing outbreak. ${ }^{[7]}$ Drugs can directly or indirectly affect intracellular Ca2 + regulation of cellular function, and Nishina $\mathrm{K}$ et al found that intravenous anesthetics may reduce neutrophil function by reducing intracellular $\mathrm{Ca} 2+$ concentration. ${ }^{[8]}$ The mechanism of action may be that intravenous anesthetics, by virtue of their high lipid solubility, accumulate on the cell membrane, change the physical structure of the membrane, and cause the membrane enzyme action, especially the inhibition of protein kinase $\mathrm{C}$, to change the cell function. In this study, two kinds of steroidal muscle relaxants, pipecuronium and vecuronium, were observed to slightly increase the expression of cell surface adhesion molecules, suggesting that steroidal muscle relaxants may change the cell membrane homeostasis, which is similar to the mechanism of action of intravenous anesthetics on the cell membrane.

In this study, only two steroidal muscle relaxants with low concentration showed inhibitory effect on CD11b expression, so it was speculated that the anti-inflammatory effect of steroidal muscle relaxants might be related to the presence of androstane structural groups. Krumholz W et al found that pancuronium bromide had an inhibitory effect on the adhesion of neutrophils in vitro, and the present study also found that vecuronium bromide and pipecuronium bromide with androstane structural groups could inhibit the expression of adhesion molecules, suggesting that the anti-inflammatory effect of muscle relaxants is related to its androstane structural groups. ${ }^{[6]}$

As a water-soluble glycosylated lipid complex, lipopolysaccharide is the main component of the cell wall of gram-negative bacteria. LPS is an important mononuclear macrophage and neutrophil strong activator, which can cause the high expression of CD11b on the neutrophil surface and lead to the formation and activation of inflammatory responses by TNF-aIIL-10IL-6IIL-8 and NO, and other pro-inflammatory factors. This experiment adopts the lipopolysaccharide as activators of whole blood in vitro, and whole blood $37^{\circ} \mathrm{C}$ in lipopolysaccharide common incubation after 2 hour, by observing and analyzing the $\mathrm{CD} 11 \mathrm{~b}$ expression of complete blood found that CD11b expression significantly higher in the surface of neutrophils, the mechanism may be related inflammatory response is a combination of a variety of channels, speculate that the main way to have: (1) LPS activated the Nuclear Factor Kappa B, launch a 
variety of inflammatory related gene transcription, inflammatory factor to further activate the surface of the PMN CD11b/CD18 expression. CD11b/CD18, through transmembrane signal transduction, promotes the activity of inflammatory transcription factor - Nuclear Factor Kappa B, inhibits cell apoptosis, and further promotes the increase of inflammatory gene expression products. These inflammatory mediators further up-regulate the expression of CD11b/CD18. LPS activated PMN, IKBa(nuclear factor of kappa light polypeptide gene enhancer in B-cells inhibitor, alpha) first significantly degraded, and then synthesis increased, inhibiting the activation effect of Nuclear Factor Kappa B, which led to a sharp increase in the initial expression of $C D 11 \mathrm{~b} / \mathrm{CD} 18$, followed by a decrease in the expression rate. (4) this test used whole blood environment, lipopolysaccharide in addition to the activation of neutrophils may also effect on macrophages and other mononuclear cells induced by producing a variety of inflammatory cell factor mutual activation of signaling pathways, before cascade amplification effect and activate CD11b expression. Induced by lipopolysaccharide CD11b express function strong, two kinds of steroid used in this test and muscle relaxants anti-inflammatory effects weak cannot restrain the effect of the activation. CD11b expression on lymphocyte surface was also observed to be stable, indicating that lipopolysaccharide did not affect lymphocyte activation, which may be related to the specificity of transcription factor-Nuclear factor-kappaB(NF-kB) or negative transcriptional feedback regulation of I B in lymphocyte, as well as other possible intermediate mechanisms.

In this study, it was found that two steroidal muscle relaxants could inhibit the expression of adhesion molecules at clinical microdose, which was consistent with the results of Krumholz W et al. 's study on pancuronium bromide. The results showed that vecuronium bromide and pipecuronium bromide had only a slight inhibitory effect on the expression of adhesion molecules on the surface of neutrophils, but could not effectively affect the activation and expression of lipopolysaccharide, and had a concentrationdependent effect.The muscle relaxants vecuronium bromide, pancuronium bromide and pipecuronium bromide have similar structures with acetyl groups, and choline receptors interact with the postsynaptic membrane by means of them. Pancuronium bromide has two functional groups. As a derivative of pancuronium bromide, vecuronium only lacks n-methyl groups at 2 positions. This structure leads to the easy hydrolysis reaction of the acetic acid root at C3 position, and the stability of the solution is lower than that of pancuronium bromide, and the action time is shorter.The structural difference between piperazine ring and pancuronium is that piperazine ring exists in the former and piperazine ring in the latter.Obviously, except for a few secondary groups, the molecular structure of the three steroidal muscle relaxants is very similar, and all of them can show some anti-inflammatory effects.

The slight inhibitory effect of steroidal muscle relaxants on neutrophil function was speculated to be related to the following mechanism by combining the results of various drugs on neutrophil adhesion. The structure of myasthenic steroids contains androstane like structural groups similar to steroid hormones, with mild anti-inflammatory and immunosuppressive functions. ${ }^{[9.10]}$ The steroids muscle relaxants may change the physical structure of the cell membrane, causing the membrane enzyme action, especially the inhibition of protein kinase $\mathrm{C}$, leading to the opening and closing of the ion channel, changing the $\mathrm{PH}$ value of intracellular acid and base, leading to changes in cell function. ${ }^{8,11,12,13]}$ 
Steroidal muscle relaxants can reduce integrin expression by inhibiting intracellular receptor signaling pathway and kinase phosphorylation. ${ }^{[14,15]}$ In addition, nitric oxide synthase is regulated to inhibit the production of nitric oxide. ${ }^{[16]}$ It shall directly or indirectly inhibit the generation of transcription factors (nf$\mathrm{kb})$ and transcription factor activated protein-1 (ap-1). ${ }^{[10,17]}$

In addition, the whole blood contains a small amount of lactoferritin (LF), which is an important immune regulator with anti-inflammatory and antioxidant properties and can reduce the release of free radicals. Neutrophil mast cells contain large amounts of LF receptors on their surface, and LF binds to receptors on the cell surface to regulate its immune function.Studies have found that LF can be expressed in mast cells and inhibit the IgE induced mast cell degranulation reaction, stabilize the inhibition of trypsin, and inhibit mast cell activation during allergy. ${ }^{[18]}$ Steroid muscle relaxants may, like glucocorticoid structures, up-regulate the expression of steroid receptor target genes and the transcription of lactoferritin, thereby inhibiting the expression of CD11b on the surface of neutrophils. ${ }^{[19]}$

In conclusion, a certain dose of vecuronium and pipecuronium may inhibit the expression of CD11b on neutrophil surface. However, the effect of high concentration of vecuronium bromide and pipecuronium bromide in this experiment was not obvious, and the concentration and test method of the drug may be closely related to its results, and the specific mechanism still needs to be studied in a large sample.

\section{Abbreviations}

PMN polymorphonuclear

CD11b macrophage differentiation antigen-1

NO nitric oxide

LPS lipopolysaccharide

FITC fluorescein isothiocyanate

PBS phosphate buffered saline

FCM flow cytometry

MCF mean fluorescence intensity

NO nitric oxide

TNF- $a$ tumor necrosis factor- $a$

LF lactoferritin 


\section{Declarations}

\section{-Ethics approval and consent to participate}

This experiment was conducted under the approval of medical ethics committee of Liaocheng people's hospital, China. All subjects involved in the study have signed informed consent and obtained consent in advance. The project has received full ethical approval and participation consent. This study has been registered in China clinical trial registry, the registration number is ChiCTR1900024761. URL of the registry: http://www.chictr.org.cn/index.aspx.

\section{-Consent to publish}

All authors reviewed and consented to the publication of the manuscript.

\section{-Availability of data and materials}

The datasets used and/or analysed during the current study available from the corresponding author on reasonable request.

\section{-Competing interests}

There is no conflict of interest with any other institution or individual. Authors without conflicts of interest, including relevant financial interests, activities, relationships, and affiliations.

\section{-Funding}

The project was funded by individual payments from the authors.

\section{-Authors' Contributions}

Zhenzhen Liu undertook the signing of informed consent and the auxiliary collection and arrangement of sample data. Ping Zhang is responsible for document retrieval and data collection. Zunyuan Liu is responsible for leading the whole process of the experimental project and writing the paper.

\section{-Acknowledgements}

Thanks for the support provided by the central laboratory of Liaocheng people's hospital in Shandong Province, China.

\section{References}

1. Krumholz W, Käbisch S, Biscoping J, Wiedemann M, Hempelmann G. [Effect of pancuronium bromide on the adherence of polymorphonuclear neutrophilic granulocytes in vitro]. Anaesthesist 
1988; 37(4):246-8.

2. Loop T, Liu Z, Humar M, Hoetzel A, Benzing A, Pahl HL, Geiger KK, JPnnen BH. Thiopental inhibits the activation of nuclear factor KB. Anesthesiology 2002; 96(5):1202-13.

3. Goto Y, O'Malley C, Fanning NF, Wang J, Redmond HP, Shorten GD: Benzodiazepines inhibit the rate of neutrophil apoptosis. Ir J Med Sci 2003; 172(4):191-4

4. Kaasaki T, Ogata M, Kawasaki C, Ogata J, Inoue Y, Shigematsu A. Ketamine suppresses proinflammatory cytokine production in human whole blood in vitro. Anesth Analg 1999; 89(3):665669.

5. Heller A, Heller S, Blecken S, Urbaschek R, Koch T. Effects of intravenous anesthetics on bacterial elimination in human blood in vitro. Acta Anaesthesiol Scand 2010; 42(5):518-26.

6. Krumholz W, Endrass J, Hempelmann G. Inhibition of phagocytosis and killing of bacteria by anaesthetic agents in vitro. Br J Anaesth 1995; 75(1):66-70.

7. Mühling J, Gonter J, Nickolaus KA, Matejec R, Welters ID, Wolff M, Sablotzki A, Engel J, Krüll M, Menges T, Fuchs M, Dehne MG, Hempelmann G. Benzodiazepine receptor-dependent modulation of neutrophil (PMN) free amino- and a-keto acid profiles or immune functions. Amino Acids 2005; 28(1):85-98.

8. Nishina K, Akamatsu H, Mikawa K, Shiga M, Maekawa N, Obara H, Niwa Y. The inhibitory effects of thiopental, midazolam, and ketamine on human neutrophil functions. Anesth Analg 1998; 86(1):15965.

9. Langereis JD, Raaijmakers HA, Ulfman LH, Koenderman L. Abrogation of NF-KB signaling in human neutrophils induces neutrophil survival through sustained p38-MAPK activation. J Leukoc Biol 2010; 88(4):655-64.

10. Crockard AD1, Boylan MT, Droogan AG, McMillan SA, Hawkins SA. Methylprednisolone induced neutrophil leukocytosis down modulation of neutrophil L-selectin and Mac-1 expression and induction of granulocyte colony stimulating factor.International Journal of Clinical \& Laboratory Research 1998; 28 (2):110-115.

11. Kanaya N, Zakhary DR, Murray PA, Damron DS. Thiopental alters contraction, intracellular Ca2+, and $\mathrm{pH}$ in rat ventricular myocytes. Anesthesiology 1998; 89: 202-214.

12. Saad MM, Eom W, Hu G, Kim SJ, Crystal GJ. Persistency and pathway of isoflurane-induced inhibition of superoxide production by neutrophils. Can J Anaesth 2010; 57(1):50-57.

13. Hollmann MW,Wieczorek KS, Berger A, Durieux ME. Local anesthetic inhibition of G protein-coupled receptor signaling by interference with Ga\r, q $\backslash r$, protein function. Mol Pharmacol 2001; 59(2):294301.

14. Ghori K, O'Driscoll J, Shorten G. The effect of midazolam on neutrophil mitogen-activated protein kinase. Eur J Anaesthesiol 2010; 27(6):562-5.

15. Kamen LA, Schlessinger J, Lowell CA. Pyk2 is required for neutrophil degranulation and host defense responses to bacterial infection. Journal of immunology (Baltimore, Md. : 1950),2011; 186(3):165665. 
16. Nelson CJ, Dykstra LA, Lysle DT. Comparison of the time course of morphine??s analgesic and immunologic effects. Anesthesia \& Analgesia 1997; 85(3):620-6.

17. Loop T, Liu Z, Humar M, Hoetzel A, Benzing A, Pahl HL, Geiger KK, J Pannen B. Thiopental inhibits the activation of nuclear factor kappaB. Anesthesiology 2002; 96(5):1202-13.

18. He $S \bowtie M c E u e n$ AR囚Blewett SA囚 Li $P$, Buckley MG , Leufkens $P$, Walls AF. The inhibition of mast cell activation by neutrophil lactoferrin: uptake by mast cells and interaction with tryptase, chymase and cathepsin G. Biochemical Pharmacology 2003;65(6):1007-1015.

19. Chua SS, Ma Z, Ngan E, Tsai SY. Cdc25B as a steroid receptor coactivator. Vitamins \& Hormones, 2004, 2004;68(6):231-56.

\section{Table 1}

Table1. Effects of samples in each group on the average optical density intensity of cd11b expression $\square, \mathrm{n}=16 \square$.

\begin{tabular}{lcc}
\hline group & Concentration The average optical density intensity of cd11b expression \\
\hline blank group & & \\
pipecuronium & 0.5 & $307.304 \pm 15.623$ \\
& 0.1 & $262.841 \pm 26.722$ \\
vecuronium & 0.5 & $331.811 \pm 92.084$ \\
& 0.1 & $268.429 \pm 5.208$ \\
LPS & & $810.672 \pm 62.232$ \\
pipecuronium+lps & 0.5 & $765.107 \pm 55.833$ \\
& 0.1 & $801.510 \pm 50.283$ \\
vecuronium+lps & 0.5 & $791.032 \pm 48.601$ \\
& 0.1 & $813.651 \pm 32.233$ \\
\hline
\end{tabular}




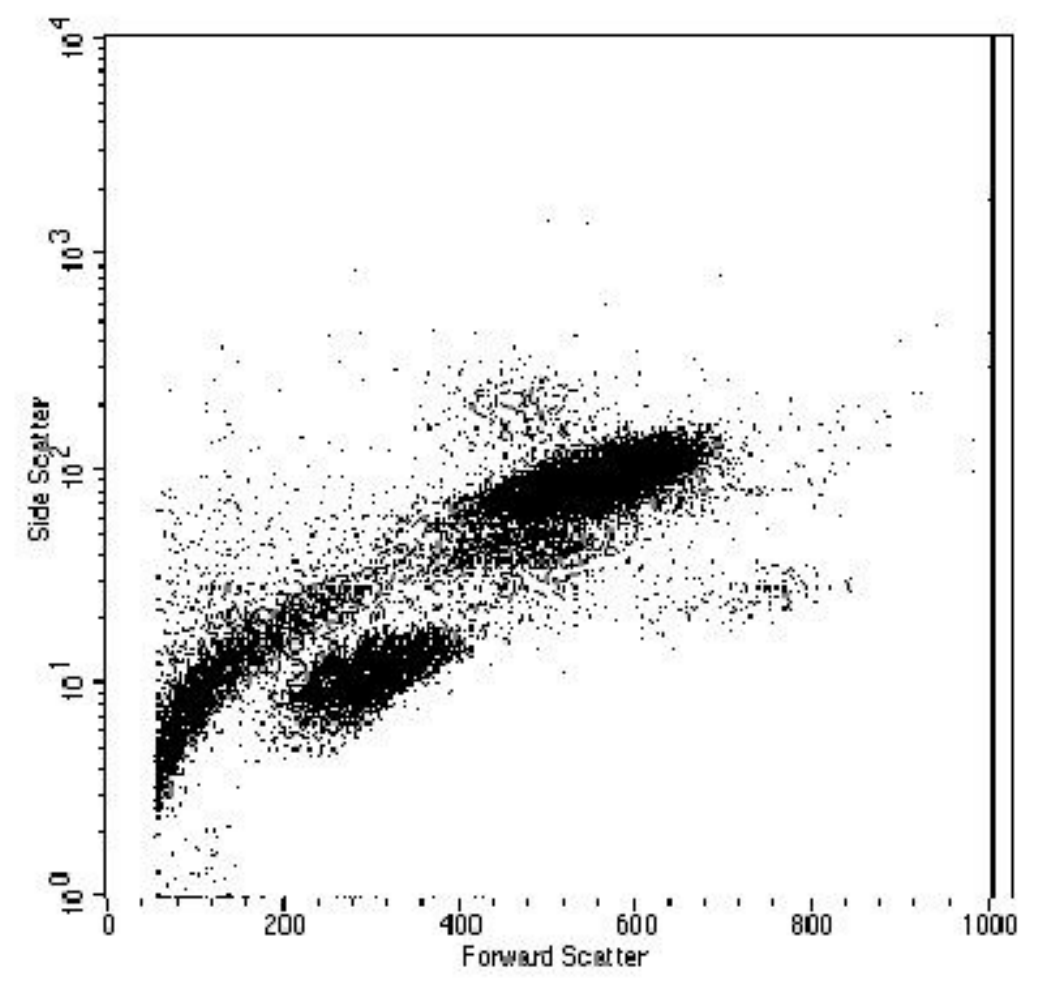

Figure 1

Optical density intensity of CD11b expression in isolated venous blood cells under resting and unstimulated conditions .

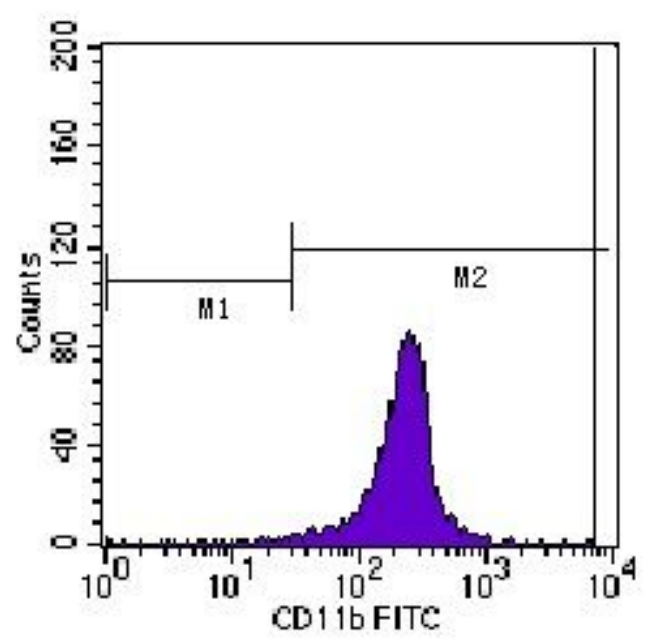

Figure 2

Expression of neutrophils $\mathrm{cd} 11 \mathrm{~b}$ in isolated venous blood of resting and unstimulated group. 


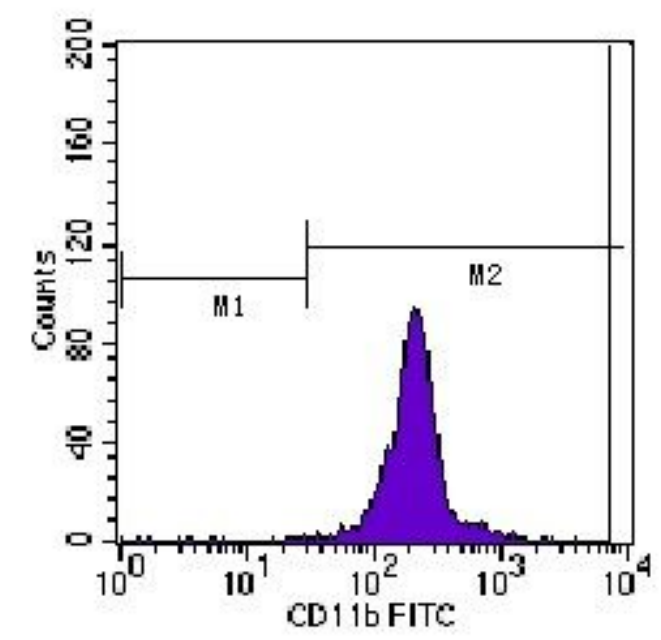

Figure 3

Expression of neutrophils CD11b in isolated venous blood of low concentration vecuronium group without stimulation.

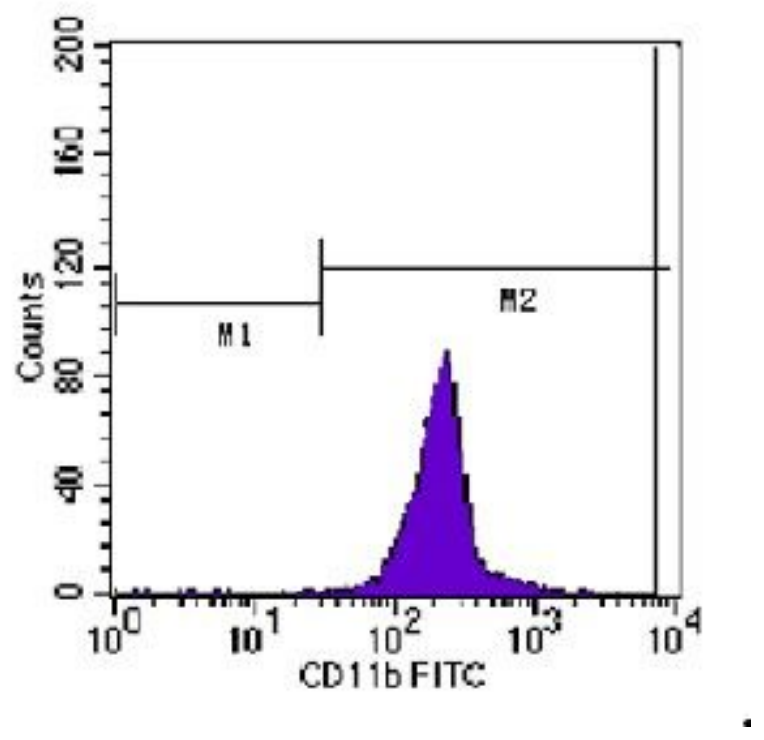

Figure 4

Expression of neutrophils CD11b in isolated venous blood of low concentration pipecuronium group without stimulation.

\section{Supplementary Files}

This is a list of supplementary files associated with this preprint. Click to download.

- CONSORTFlowDiagra.docx 